\title{
Central venous catheter-related superior vena cava syndrome following renal transplantation -A case report-
}

\author{
Misook Seo, Won-Jung Shin, and In-Gu Jun \\ Department of Anesthesiology and Pain Medicine, Asan Medical Center, University of Ulsan College of Medicine, Seoul, Korea
}

A 55-year-old man with end-stage renal disease had severe left ventricular dysfunction and a history of deep vein thrombosis. He underwent renal transplantation, during which a central venous catheter was inserted into the right jugular vein. The central venous pressure (CVP) exceeded $20 \mathrm{mmHg}$ throughout the operation but there was no other adverse event. After surgery, although the left ventricular dysfunction improved, the CVP remained high. On postoperative day 10, the patient presented with cyanosis of the arms and redness of the face and was diagnosed with superior vena cava (SVC) syndrome, for which he underwent emergency thrombectomy and SVC reconstruction. The clinical course of this patient suggests that his end-stage renal disease-associated hypercoagulable state may have promoted thrombus formation. Moreover, placing the central venous catheter tip too deep may have encouraged thrombus formation. Repositioning the tip may have prevented this complication. (Korean J Anesthesiol 2012; 63: 550-554)

Key Words: Central venous catheter, Renal transplantation, Superior vena cava syndrome, Thrombus.

A central venous catheter (CVC) is an intravenous line that is widely used to monitor patients intra-operatively. This vascular access device is used to measure hemodynamic variables and to administer large volumes of blood, fluids, medications and nutrition that cannot be delivered safely through peripheral venous catheters. However, about $15 \%$ of patients have been reported to experience adverse events following catheter insertion, including infection, hematoma, thrombosis, and pneumothorax $[1,2]$. In particular, thrombotic complications occur in $2 \%$ to $21 \%$ of patients depending on the insertion site [2]. This relatively high thrombotic complication incidence may be related to use various diagnostic techniques, venography and ultrasonography, without clinical symptoms which usually present with total occlusion. CVC-related thrombosis can result in superior vena cava (SVC) syndrome, a potentially fatal clinical manifestation of SVC obstruction that is characterized by a severe reduction in the venous return from the head, neck, and upper extremities [3-5]. Here, the case of a patient with end-

Received: November 2, 2011. Revised: January 15, 2012. Accepted: January 30, 2012.

Corresponding author: In-Gu Jun, M.D., Department of Anesthesiology and Pain Medicine, Asan Medical Center, University of Ulsan College of Medicine, 388-1, Pungnap-2dong, Songpa-gu, Seoul 138-040, Korea. Tel: 82-2-3010-3868, Fax: 82-2-470-1363, E-mail: nicezizon@naver.com (c)This is an open-access article distributed under the terms of the Creative Commons Attribution Non-Commercial License (http:// creativecommons.org/licenses/by-nc/3.0/), which permits unrestricted non-commercial use, distribution, and reproduction in any medium, provided the original work is properly cited. 
stage renal disease (ESRD) who experienced SVC syndrome after CVC placement in the right internal jugular vein for renal transplantation is described.

\section{Case Report}

A 55-year-old man who developed ESRD secondary to type II diabetes mellitus nephropathy underwent living related renal transplantation. He had been diagnosed with diabetes mellitus 10 years earlier and had been on hemodialysis for 3 years. Due to a history of deep vein thrombosis and left arm arteriovenous fistula thrombosis, he had been taking the antithrombotic agents warfarin and clopidogrel. However, he elected to stop taking these medications 10 days before transplantation. Preoperative laboratory tests showed a BUN of $30 \mathrm{mg} / \mathrm{dl}$, a serum creatinine concentration of $6.2 \mathrm{mg} / \mathrm{dl}$, a potassium concentration of $5.0 \mathrm{mmol} / \mathrm{L}$, a serum cholesterol concentration of $141 \mathrm{mg} / \mathrm{dl}$, a prothrombin time (PT) of 1.17 PT-INR (international normalized ratio), an active partial thromboplastin time (aPTT) of 29.9 seconds, and a serum cholesterol concentration of $141 \mathrm{mg} / \mathrm{dl}$. Chest X-rays showed no active lesions in either lung field. Preoperative transthoracic echocardiography revealed severe left ventricular dysfunction, with an ejection fraction of $24 \%$, which was controlled by digoxin.

The patient did not receive premedication on the day of surgery. Before anesthesia was induced, his radial artery was cannulated with local anesthesia for arterial pressure monitoring. General anesthesia was induced with IV lidocaine (40 mg), etomidate (12 mg), and atracurium (50 mg). After tracheal intubation, anesthesia was maintained with $6-7 \%$ desflurane in $50 \%$ oxygen with $\mathrm{N}_{2} \mathrm{O}$. To monitor central venous pressure (CVP) and to infuse drugs and fluids, a CVC (PreSep ${ }^{\mathrm{TM}}$, Edwards Lifesciences, Irvine, CA, USA) was inserted into his right internal jugular vein. Cannulation was performed under ultrasound guidance. A venous valve or thrombus was not visible in his right jugular vein. The CVC was successfully inserted during the first trial without complications. The function of the CVC was checked, and the catheter was fixed 15 $\mathrm{cm}$ from the tip. During surgery, the CVP of the patient ranged from $20 \mathrm{mmHg}$ to $25 \mathrm{mmHg}$, but his other vital signs remained stable and the CVP wave form was normal. His total urine output after reperfusion was $180 \mathrm{ml}$. The patient was transferred to the intensive care unit at the end of the operation. On the day after the operation, his CVP stayed between $20 \mathrm{mmHg}$ and 25 $\mathrm{mmHg}$, and his urine output was more than 3,000 $\mathrm{ml}$ per day. PT and aPTT were 1.03 PT-INR and 30.8 seconds, respectively. On postoperative day 3 , his CVP increased to $30 \mathrm{mmHg}$, but the patient had no complaints and was moved to the general ward. On postoperative day 8 , his CVP increased further to
$38 \mathrm{mmHg}$ and his urine output decreased to below 1,300 ml per day. An emergency echocardiogram showed that his left ventricular ejection fraction was $55 \%$. A cardiologist judged that the preoperative heart failure of the patient had improved and was not the cause of his elevated CVP. The patency of the CVC lumen had been checked daily up until that point, but problems had never been observed. On postoperative day 9, the patient complained of dizziness and presented with facial redness, cyanosis of the upper extremities, and right neck vein engorgement. He was assessed for SVC syndrome, followed by an urgent angiographic computed tomography (CT) scan. CT images revealed diffuse low attenuation around the central line within the distal internal jugular vein and proximal SVC, suggesting a thrombus (Fig. 1). X-rays that had been taken on post-transplantation days 1 (Fig. 2) and 8 (Fig. 3) were then evaluated retrospectively and compared. A slight difference was observed near the neck: on the day 8 X-ray, the CVC shape had become bent near the proximal right internal jugular vein. On postoperative day 10, the patient was diagnosed with SVC syndrome resulting from a CVC thrombus. Since the thrombus was considered to be too big to remove with anticoagulation therapy, he underwent emergency surgery to remove the thrombus. Anesthesia was induced with etomidate (10 mg) and atracurium (10 mg) and maintained with propofol at $1 \mathrm{mcg} /$ $\mathrm{kg} / \mathrm{min}$ and remifentanil at $15 \mathrm{ng} / \mathrm{kg} / \mathrm{min}$. To monitor his vital signs, a new arterial line and CVC were inserted into his femoral artery and vein. Cardiac surgeons removed the CVC and thrombus in his right internal jugular vein and reconstructed the SVC-right atrium junction with bovine pericardium using

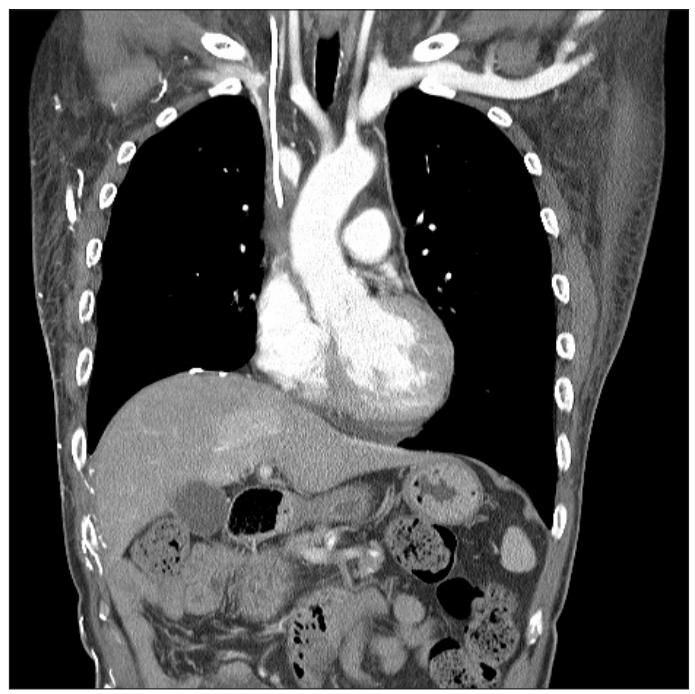

Fig. 1. CT image showing diffuse low attenuation around the central line within the distal internal jugular and proximal superior vena cava (SVC) that was suggestive of a thrombus. 


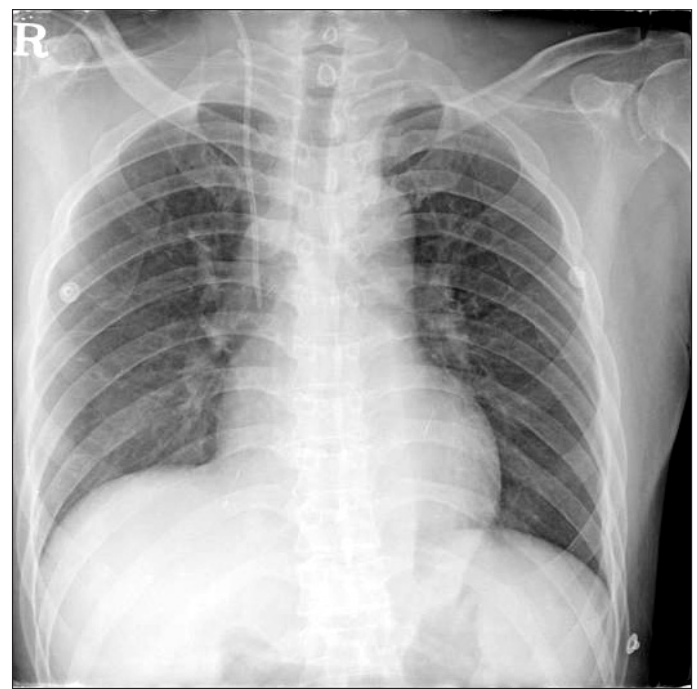

Fig. 2. Chest X-ray 1 day after renal transplantation. The tip of the central venous catheter (CVC) was below the carina.

a cardiopulmonary bypass. On post-thrombectomy day 4 , he was extubated and moved to the general ward. His symptoms, including the facial redness and edema, disappeared and his urine output was maintained at over 2,000 $\mathrm{ml}$ per day. $\mathrm{He}$ was restarted on oral anticoagulation with warfarin on postthrombectomy day 5 . On post-thrombectomy day 17 , the patient was discharged without sequelae.

\section{Discussion}

Mechanical complications related to central venous catheterization have been reported in $5 \%$ to $19 \%$ of patients who receive CVC. Although arterial puncture, hematoma and pneumothorax are more common than other complications, thrombus formation remains a major complication of CVC insertion [1]. The risk of catheter-related thrombosis varies depending on the site of insertion, with one study reporting that the risk was approximately 4 -fold higher if the insertion site was the internal jugular vein rather than the subclavian vein [2]. SVC syndrome is rarely induced by CVC-related thrombosis. Rather, malignant tumors are the leading cause of SVC syndrome. However, due to the increased use of CVC, other causes have become more common.

The SVC is the main vessel that collects the venous stream from the upper extremities, head, and upper torso and brings it to the heart. As the SVC becomes compressed, the venous blood flow resistance increases, causing the symptoms and signs of SVC syndrome, the most frequent being neck and facial swelling, dyspnea, and cough. Other symptoms include

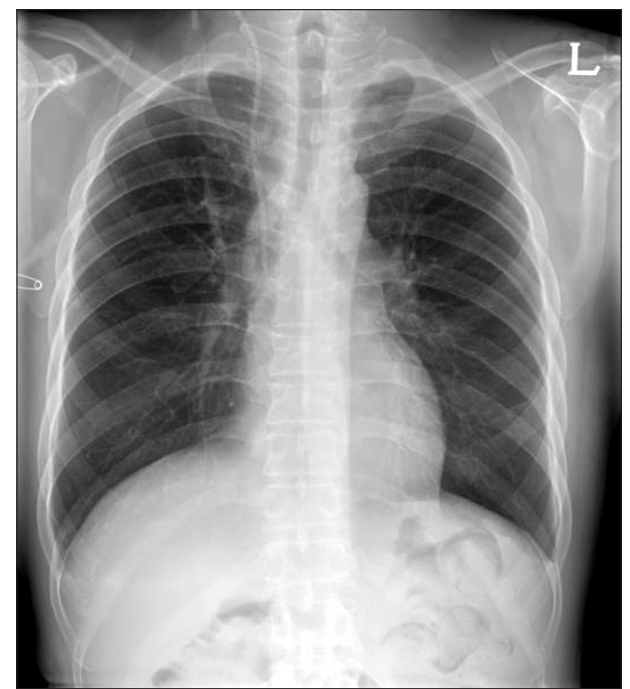

Fig. 3. Chest X-ray 8 days after renal transplantation. Compared to the day $1 \mathrm{X}$-ray, the central venous catheter (CVC) shape had become bent near the proximal right internal jugular vein.

hoarseness, headaches, nasal congestion, tongue swelling, dizziness, pain, hemoptysis, epistaxis, and syncope, with physical findings including cyanosis, dilated neck veins, and edema of the face, arms, and chest [4,6]. Management options for patients with SVC syndrome include treatment of the original malignancy, endovascular stenting, anticoagulation, and surgery [4].

Catheter tip position is a risk factor for thrombosis: placing the tip in the brachiocephalic vein and the upper third of the SVC results in a 3 - to 5 -fold increase in thrombotic complications [7]. However, placing the CVC tip below the SVCright atrium (RA) junction or in the RA increases the risk of serious complications such as cardiac tamponade and RA rupture. To prevent such life-threatening complications and to reduce the incidence of thrombosis, the CVC tip should be located within the SVC-RA junction [7-10]. However, it can be difficult to identify this junction. The carina can be a landmark of CVC depth in standard posterior-anterior chest radiography. A study with fresh cadavers found that the carina was located $0.8 \mathrm{~cm}$ above the pericardial sac [10]. Thus, for reasons of safety, the tip of the catheter should not be below the carina $[10,11]$. To determine the length of a CVC that is most adequate, it is useful to measure the distance from the insertion site to the ipsilateral clavicular notch at the bedside in addition to measuring the length from the notch to the carina on the chest X-ray [12]. The tip location should be confirmed by postoperative X-rays and can be repositioned if necessary. In our patient, the CVC tip was located below the carina and within the cardiac shadow. Fortunately, there were no serious complications such as 
cardiac tamponade, but it would have been safer to reposition the CVC. Although a tip position higher than the SVC-RA junction increases the risk of thrombotic complication, the results in our patient indicate that thrombosis can also occur when a lower tip position is used.

Catheter-associated variables, such as size, time, tip location, and types of infusion, influence the risk of thrombosis. For example, the risk of thrombus is greater with a large-diameter catheter that prevents blood flow than with a smaller catheter [8]. Thrombus formation can also be triggered by catheterinduced vessel wall trauma. The coagulation pathway is activated, and persistent inflammation due to the presence of the catheter can induce intimal hyperplasia [13].

In our patient, the CVC was maintained for more than one week. After thrombectomy was performed, a retrospective comparison of the day 1 and 8 post-transplantation X-rays revealed a difference that was thought to indicate the thrombus in the internal jugular vein, namely a bend in the CVC shape near the proximal right internal jugular vein. Thus, although the thrombus could not be diagnosed directly by X-rays, the X-rays nevertheless still indicated signs of the thrombus before its presence was verified by CT.

Patients with ESRD have several factors, such as diabetes, obesity, cancer, and major surgery, which predispose them to an increased risk of venous thromboembolism [14]. Although the relationship between ESRD and venous thromboembolism is unclear, patients with ESRD usually have elevated concentrations of fibrinogen and inflammatory markers. In addition, endothelial cell dysfunction, enhanced platelet activation and aggregation, hypercholesterolemia, hypertriglyceridemia, and activation of the coagulation system are considered to be factors that increase the risk of venous thromboembolism in patients with ESRD $[14,15]$. Other epidemiological studies have reported that patients undergoing dialysis and renal transplant recipients are at increased risk of venous thromboembolism [14]. Moreover, the enhanced hemostatic thromboelastograph findings in patients with ESRD are relieved after renal transplantation along with corrections in their hypertriglyceridemia and uremia [15]. With regard to our patient, he had received warfarin therapy before transplantation due to his previous history of deep vein thrombosis and arteriovenous thrombosis. However, he had elected to stop taking prophylactic anticoagulation 10 days before renal transplantation and was not restarted on these agents after the operation because the transplant surgeon was concerned about post-operative bleeding. Since stopping anticoagulation could induce thrombotic complication, it may have been beneficial for this patient if anticoagulation had been restarted early in the postoperative period.

The CVP of our patient was at high levels during the trans- plantation surgery. This was considered to reflect heart failure because the CVC wave form was normal. Later, it was judged that the heart failure of the patient had improved after surgery and it was then realized that it was new thrombus formation that kept the CVP at high levels in the postoperative period.

In summary, a renal transplantation recipient with ESRD whose blood was in a hypercoagulable state experienced potentially fatal CVC-related thrombotic complications. For such patients, the risk of thrombus formation may be reduced by carefully determining the CVC depth before insertion on the basis of preoperative X-rays by repositioning the tip on the basis of postoperative X-rays and by supplying appropriate anticoagulation early in the postoperative period.

\section{References}

1. McGee DC, Gould MK. Preventing complications of central venous catheterization. N Engl J Med 2003; 348: 1123-33.

2. Merrer J, De Jonghe B, Golliot F, Lefrant JY, Raffy B, Barre E, et al. Complications of femoral and subclavian venous catheterization in critically ill patients: a randomized controlled trial. JAMA 2001; 286: 700-7.

3. Di Cocco P, Orlando G, Di Cesare E, Mazzotta C, Rizza V, Pisani F, et al. Superior vena cava syndrome due to thrombotic occlusion in a thrombophilic renal transplant recipient: a case report. Transplantation Proc 2010; 42: 1358-61.

4. Wan JF, Bezjak A. Superior vena cava syndrome. Emerg Med Clin North Am 2009; 27: 243-55.

5. Ruesch S, Walder B, Tramer MR. Complications of central venous catheters: internal jugular versus subclavian access--a systematic review. Crit Care Med 2002; 30: 454-60.

6. Abner A. Approach to the patient who presents with superior vena cava obstruction. Chest 1993; 103(4 Suppl): S394-7.

7. Caers J, Fontaine C, Vinh-Hung V, De Mey J, Ponnet G, Oost C, et al. Catheter tip position as a risk factor for thrombosis associated with the use of subcutaneous infusion ports. Support Care Cancer 2005; 13: $325-31$.

8. Eastridge BJ, Lefor AT. Complications of indwelling venous access devices in cancer patients. J Clin Oncol 1995; 13: 233-8.

9. Cadman A, Lawrance JA, Fitzsimmons L, Spencer-Shaw A, Swindell $R$. To clot or not to clot? That is the question in central venous catheters. Clin Radiol 2004; 59: 349-55.

10. Albrecht K, Nave H, Breitmeier D, Panning B, Tröger HD. Applied anatomy of the superior vena cava-the carina as a landmark to guide central venous catheter placement. Br J Anaesth 2004; 92: 757 .

11. Yoon SZ, Shin JH, Hahn S, Oh AY, Kim HS, Kim SD, et al. Usefulness of the carina as a radiographic landmark for central venous catheter placement in paediatric patients. Br J Anaesth 2005; 95: 514-7.

12. Kim MC, Kim KS, Choi YK, Kim DS, Kwon MI, Sung JK, et al. An estimation of right- and left-sided central venous catheter insertion depth using measurement of surface landmarks along the course of central veins. Anesth Analg 2011; 112: 1371-4.

13. Manderson JA, Campbell GR. Venous response to endothelial 
denudation. Pathology 1986; 18: 77-87.

14. Wattanakit K, Cushman M, Stehman-Breen C, Heckbert SR, Folsom AR. Chronic kidney disease increases risk for venous thromboembolism. J Am Soc Nephrol 2008; 19: 135-40.
15. Lee JM, Kim ES, Park CS, Kang SH. Changes in thromboelastographic findings after renal transplantation in patients with chronic renal failure. Korean J Anesthesiol 2004; 47: 75-81. 